trimethoprim/sulfa-methoxazole (77.6\%) were the lowest. All Enterococcus spp. isolates were susceptible to linezolid and vancomycin, resistan-ce ratios aganist nitrofurantoin, penicillin, and erythromycin were $5.6 \%, 19.0 \%$, and $27.3 \%$ respectively.

Although antibiotic susceptibility rates among our hospital's patient population were higher than other studies, it should be remembered that culture and antimicrobial susceptibility testing may be beneficial to help choosing narrow-spectrum antibiotics and to prevent development of resistance.

\section{PO-0800 BACTERIA ISOLATED FROM THE URINE SAMPLES OF CHILDREN AND THEIR ANTIBIOTIC SUSCEPTIBILITIES}

Y Taskiran. Çocuk, Aydin Kadin Dogum Hastanesi, Aydin, Turkey

10.1136/archdischild-2014-307384.1435

The aim of this study was to identify the bacteria isolated from urine samples and to investigate their antibiotic suscep-tibilities in children aged $0-14$ years.

Urine samples of 1820 patients who referred to our hospital and had urinary tract infection symptoms were studied in order to evaluate culture and antibiotic susceptibility testing results. All urine samples were inoculated on eosin methylene blue (EMB) and $5 \%$ blood agars. Bacterial isolates were identified by conventional met-hods. Antibiotic susceptibility testing was performed by disk diffusion method.

The bacteria were isolated in 198 (9.2\%) of 1820 urine samples. The most frequently isolated bacteria (69.4\%) were Escherichia coli. Enterococcus spp. (12.7\%), Proteus spp. (6.1\%), Klebsiella spp. (4.3\%), Enterobacter spp. (5.9\%) and coagulasenegative staphylococci (CNS) (1.5\%) followed respectively. While all E.coli, Proteus spp., Klebsiella spp., and Enterobacter spp. isolates were susceptible to amikacin, gentamicin, and imipenem, sensitivity of the ampicillin (68.5\%), and the trimethoprim/sulfa-methoxazole $(77.6 \%)$ were the lowest. All Enterococcus spp. isolates were susceptible to linezolid and vancomycin, resistan-ce ratios aganist nitrofurantoin, penicillin, and erythromycin were $5.6 \%, 19.0 \%$, and $27.3 \%$ respectively.

Although antibiotic susceptibility rates among our hospital's patient population were higher than other studies, it should be remembered that culture and antimicrobial susceptibility testing may be beneficial to help choosing narrow-spectrum antibiotics and to prevent development of resistance.

\section{Neurology and Developmental Paediatrics}

\section{PO-0801 CLINICAL AND PATHOGENETIC ASPECTS OF THE DELAYED PSYCHOVERBAL DEVELOPMENT IN CHILDREN OF AN EARLY AGE}

AG Chernykh, SB Berezhanskaya, Al Bespalova, TV Prosvetova, AA Afonin. Pediatric Department, Rostov Scientific-Research Institute of Obstetrics and Pediatrics, Rostov-onDon, Russia

\subsection{6/archdischild-2014-307384.1436}

26 children at the age of 1.5 to 4 with delayed psychoverbal development (DPVD) without movement disorders were examined. All children were born full-term, had cerebral ischaemia of the 1 st and 2 nd degrees, were observed by a neurologist during the 1 st year of life and were further observed according to their place of residence.
The complex of examination included: video-EEG monitoring of the daytime sleep, brain MRI, transcranial dopplerography (TCDG) and consultation of a psychologist, logopedist, endocrinologist and geneticist.

The data of psychological testing revealed mental retardation of a different level and character: immaturity of emotional regulation, skills of communicative behaviour and cooperative activity as well as partial delay of cognitive development against the background of general speech underdevelopment, delay of sensorimotor development (12), intellectual deficiency of cerebralorganic genesis with neurodynamic disorders (10) and traits of autistic behaviour (4).

As a result of logopedist's examination it was revealed that more than $1 / 3$ of children had alalia, 2 children had dysarthria and 15 children had dyslalia.

According to the data of video-EEG 13 children had decrease of functional lability of cortical processes, 6 children had paroxysmal activity, 2 children had epiactivity in the form of 'peakslow wave' complexes, 5 children had delay in the basic rhythm formation that conformed to the presence of organicity as per the data of brain MRI in the form of periventricular gliosis and allowed to consider DPVD of cerebral-organic type.

As a result of complex examination the following clinical entities were revealed: subclinical hypothyroidism (2), syndrome of cognitive epileptiform disintegration (2), autism (3), organic lesion of central nervous system (5), encephalasthenia (7) and syndrome of minimal brain dysfunction (9).

The analysis of results revealed different variants of DPVD: dysontogenetic variant due to immaturity, encephalopathic variant against the background of minor organic lesion of CNS, secondary DPVD against the background of hypothyroidism that determined the initial approach and amount of therapy as well as further prognosis for the disease.

\section{PO-0802 A RARE CASE OF H1N1 TRIGGERED RECURRENT ACUTE NECROTIZING ENCEPHALOPATHY (ANE1) IN A FAMILIAL COHORT WITH A T653I MUTATION IN THE RANBP2 GENE}

${ }^{1} \mathrm{G}$ Anand, ${ }^{1} \mathrm{~V}$ Ravindran, ${ }^{2} \mathrm{~F}$ Sherrin, ${ }^{3} \mathrm{~A}$ Nemeth, ${ }^{1} \mathrm{~S}$ Jayawant. ${ }^{1}$ Paediatric Neurology, Oxford University Hospitals NHS Trust, Oxford, UK; ${ }^{2}$ Paediatric Neuroradiology, Oxford University Hospitals NHS Trust, Oxford, UK; ${ }^{3}$ Paediatric Genetics, Oxford University Hospitals NHS Trust, Oxford, UK

\subsection{6/archdischild-2014-307384.1437}

Acute necrotizing encephalopathy (ANE) is a host-mediated phenomenon with viral triggers typically including influenza and parainfluenza viruses [1]. It presents with a spectrum of symptoms from vomiting to seizures and coma with a potential to cause long-standing neurocognitive impairment [2]. Sporadic (ANE) and recurrent/familial (ANE1) forms are now recognised in the literature with the latter being described after the discovery of mutations in the RANBP2 gene [1]. The diagnosis requires exclusion of other causes of encephalopathy, a high index of suspicion from the history (i.e. of recurrent attacks) and diagnostic clues from prompt MRI imaging.

Although the association of the H1N1 strain of influenza as a trigger for isolated ANE has been previously described, this is the first case of the H1N1 strain being isolated in the recurrent/ familial form, ANE1, with a confirmed missense mutation in RANBP2. This is only the second report of the T653i mutation in RANBP2 giving rise to a clinical phenotype of ANE1. In the previous reported case, the pathogenic significance of this 